\title{
Flight Test of the Quadrotor based on Algorithm of Multi-sensor Fusion
}

\author{
Lingyu Liu, ${ }^{1, a}$, Xinhua Wang ${ }^{2, b}$, Qingyan Tan ${ }^{3, b}$ \\ ${ }^{1}$ Nanjing University of Aeronautics and Astronautics, China \\ ${ }^{2}$ Nanjing University of Aeronautics and Astronautics, China \\ ${ }^{3}$ Nanjing University of Aeronautics and Astronautics, China

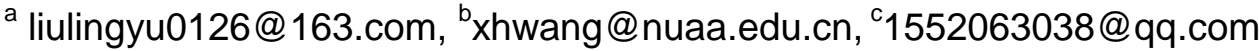

Keywords: Kalman filter; integrated navigation system; flight test

\begin{abstract}
This paper introduces a multi-sensor fusion technology and verify Whether the integrated navigation system is valid. IMU and GPS/ barometer is complementary, the former's long-term stability is poor and short-term stability is good, and the latter is just the opposite. Therefore, the fusion algorithm can be used to combine the three data, the output of three-dimensional position information, speed information, attitude information, to provide navigation information for quadrotor. In loiter mode, fluctuations of roll channel and elevation channel are reduced, height tracking situation is better .
\end{abstract}

\section{Introduction}

With the development of quadrotor technology, the function of the quadrotor is becoming more and more complete, the quadrotor needs a low cost, light quality, high precision navigation system . IMU can output high precision attitude and acceleration information, ordinary GPS can provide three-dimensional position, speed information but their precision is limited, and its anti disturbance ability is poor, and the dynamic tracking performance is generally low. Barometer can provide the relative altitude of quadrotor, but its accuracy is low and dynamic tracking performance is generally low. IMU and GPS/ barometer is complementary, the former's long-term stability is poor and short-term stability is good, and the latter is just the opposite ${ }^{[1][2][3]}$. Therefore, the fusion algorithm can be used to combine the three data, the output of three-dimensional position information, speed information, attitude information, to provide navigation information for quadrotor.

A fusion algorithm based on Kalman filter is introduced in this paper. And the integrated navigation system is used in actual flight .The result shows that In loiter mode, fluctuations of roll channel and elevation channel are reduced, height tracking situation is better. In auto mode, situation of tracking route is better.

\section{.2 multiple sensor information fusion}

The data fusion algorithm is improved on the principle of Calman filter ${ }^{[4]}{ }^{[5]}$. Calman filter is the optimal estimation of the system state through the input and output observation data. In this paper, Calman filter is applied to the discrete linear time varying system, and the linear stochastic differential equation can be described as:

$$
\begin{aligned}
& x(k)=A x(k-1)+B u(k)+w(k) \\
& y(k)=C_{X}(k)+v(k)
\end{aligned}
$$

where, $x(k)$ is the system state of $\mathrm{K}$ time, $x(k-1)$ is the optimal state of the results, $u(k)$ is the control of the system, $y(k)$ is the K time measurement, $\mathrm{w}(\mathrm{k})$ and $\mathrm{V}(\mathrm{k})$ are the noise of system process and measurement, their COVERIANCE are expressed by R and Q (assuming that the system does not change with the system state), respectively, A, B, C mean the system state parameters, system input control parameters and measurement system parameters respectively. 
System for predicting the next state of the system using the process model of the system. Assuming that the system state is $\mathrm{k}$, according to the system model, the state of the system can be predicted according to the state of the system:

$$
x(k \mid k-1)=A x(k-1 \mid k-1)+B u(k)+{ }_{W}(k)
$$

${ }_{x}(k \mid k-1)$ is to use the results of the last state prediction, $x(k-1 \mid k-1)$ is the result of the optimal state, $P(k \mid k-1)$ is the coveriance of $x(k \mid k-1)$ :

$$
P(k \mid k-1)=A P(k-1 \mid k-1) A^{T}+Q
$$

Where $P(k-1 \mid k-1)$ is the covariance of $x(k-1 \mid k-1)$. By combining the predictive value and the measurement, the optimal estimation value $x(k \mid k)$ can be obtained for the present state $\mathrm{K}$ time.:

$$
\begin{gathered}
x(k \mid k)=x(k \mid k-1)+K_{g}(k)\left(y(k)-C_{X}(k \mid k-1)\right) \\
K_{g}(k)=\frac{P(k \mid k-1) C^{T}}{C P(k \mid k-1) C^{T}+R}
\end{gathered}
$$

$K_{g}(k)$ is the Calman gain of $\mathrm{K}$ time, covariance of $x(k \mid k)$ in $\mathrm{K}$ time is:

$$
P(k \mid k)=\left(I-K_{g}(k) C\right) P(k \mid k-1)
$$

Where, I is unit matrix 。There is first-order system in this paper, Combined with the specific circumstances of the GPS/imu/ barometer, the following formula can be generated:

$$
\begin{aligned}
& x(k \mid k-1)=x(k-1 \mid k-1)+u(k) \\
& P(k \mid k-1)=P(k-1 \mid k-1)+Q \\
& K_{g}(k)=\frac{P(k \mid k-1)}{P(k \mid k-1)+R} \\
& x(k \mid k)=x(k \mid k-1)+K_{g}(k) *(y(k)-x(k \mid k-1))
\end{aligned}
$$

Where, $x(k-1 \mid k-1)$ is the optimal position or velocity for the last forecast, $u(k)$ is the estimated position or velocity from attitude reference system, $Q$ is the covariance of $u(k), \quad \mathrm{R}$ is the covariance of $y(k), K_{g}(k)$ is the Kalman gain, $y(k)$ is the position or the difference in position by the barometer/GPS., $x(k \mid k)$ is the position or velocity after fusion.

barometer sensor output height information, IMU output three axis acceleration and Euler angle, GPS output latitude and longitude and speed, Firstly, the latitude and longitude are transformed to the geodetic coordinate system with home point as the origin by the following formula:

$$
\begin{aligned}
& \text { position }_{N}=R *\left(\text { lat }_{i}-1 a t_{o}\right) * \frac{\pi}{180} \\
& \text { position }_{E}=R *\left(\text { lon }_{i}-\text { lon }_{o}\right) * \frac{\pi}{180} * \cos \left(\text { lat }_{i} * \frac{\pi}{180}\right)
\end{aligned}
$$

Where, $\mathrm{R}$ is the radius of the curvature of the earth, $10 n_{0}$ and $l a t_{o}$ are the latitude and longitude of the origin。

Then the position information and velocity information and the attitude sensor information are according to the formula for fusion, the specific process as shown below, by adjusting the value of Q and $\mathrm{R}$, you can adjust the fusion effect: 


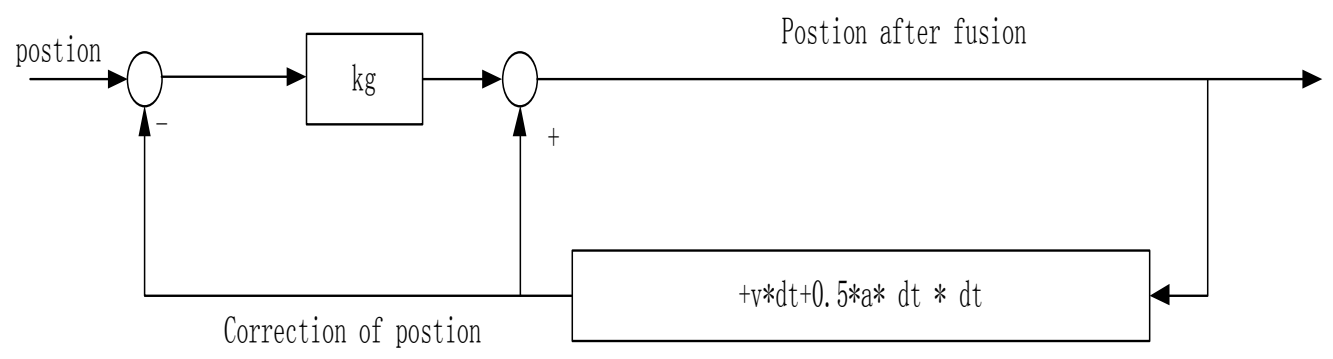

Figure 1. The processes of position's fusion

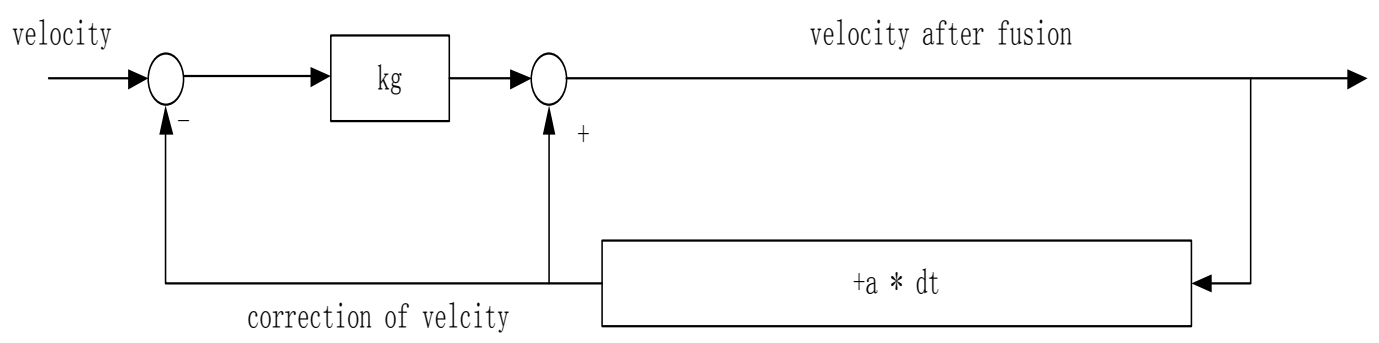

Figure 2. The processes of velocity's fusion

\section{3 flight test of quadrotor}

Position information and velocity information play a very important role in the four rotor flight. The loiter mode control as shown in the figure3, the loiter mode control by controlling the yaw and roll angle, altitude mode control as shown in the figure4.If the position, velocity information that sensors provide is not accurate, quadrotor will not hover at a fixed altitude.

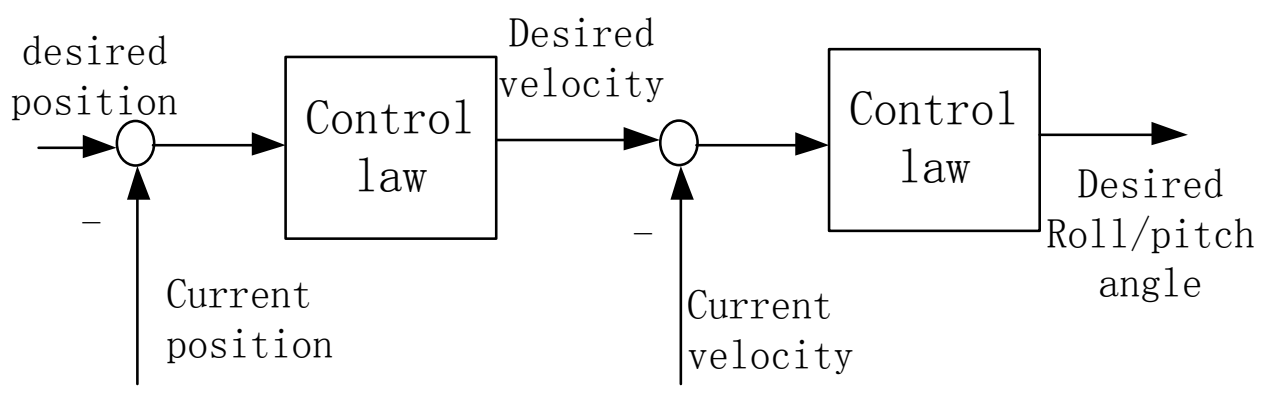

Figure 3. The processes of loiter mode control 


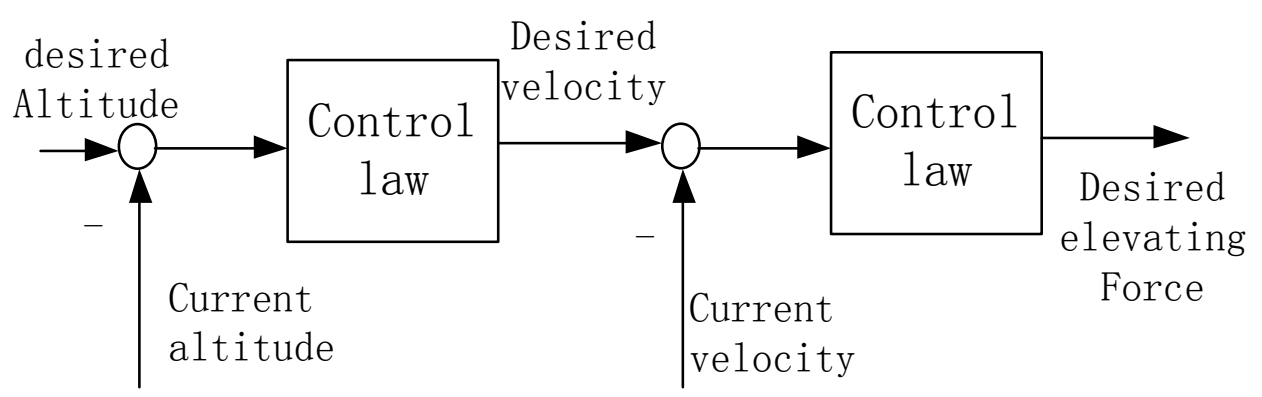

Figure 4 The processes of altitude mode control

\section{4 the results and conclusions of the experiment}

\subsection{Multi sensor fusion result}

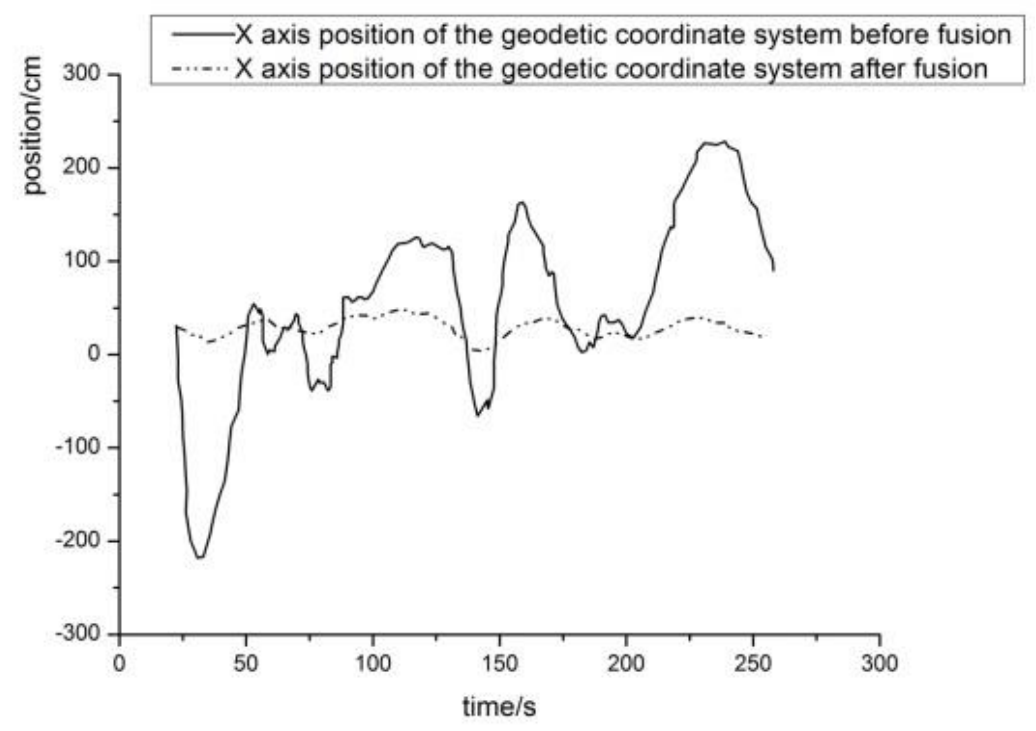

Figure 5. The result of $X$ axis position's fusion 


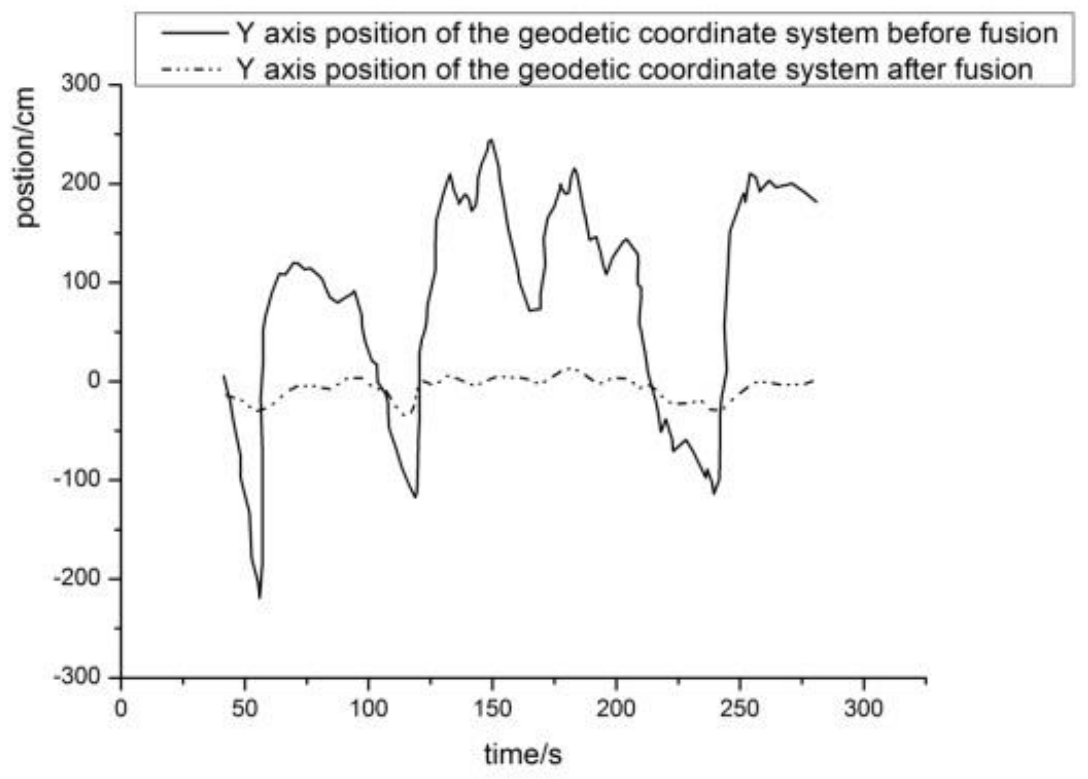

Figure 6. The result of Y axis position's fusion

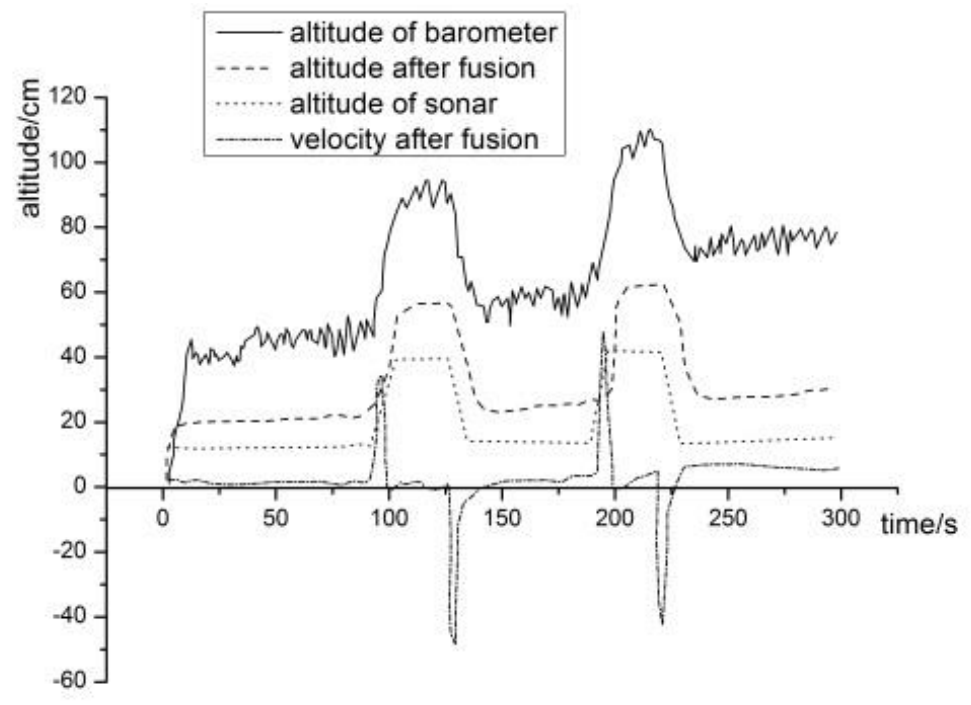

Figure 7. The result of altitude's fusion

Figure 5 and figure 6 are the fusion results of postion of gps, which show that fusion can effectively restrain the drift of position information. Figure7 is altitude fusion result, the sonar altitude is the most highly accurate data. what can be seen from the figure is that the altitude after fusion is more close to sonar altitude compared with the original data, and the speed information is consistent with the actual data. 


\section{2 flight test of quadrotor}
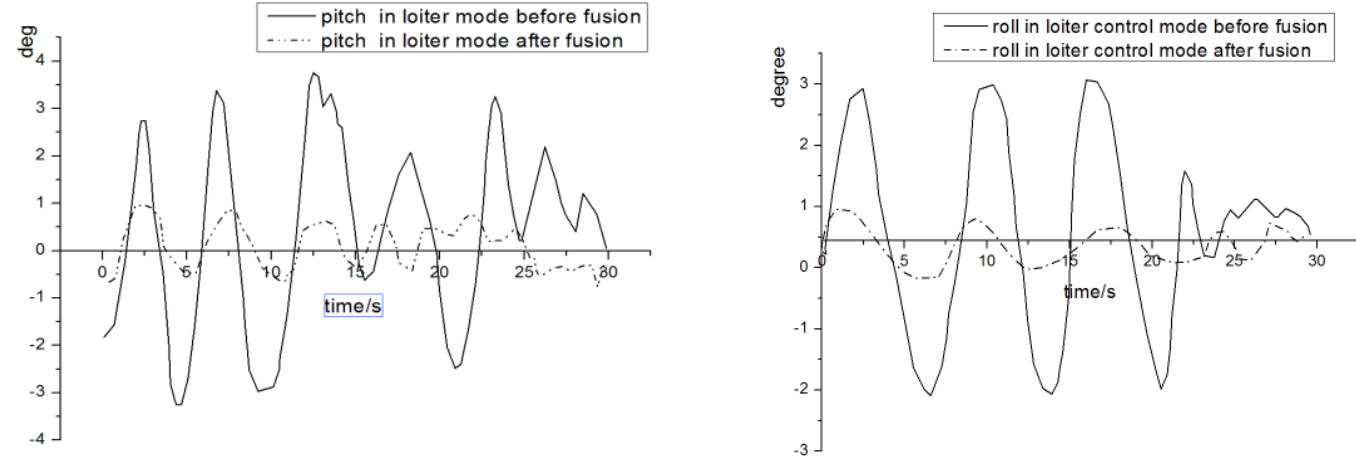

Figure 8. pitch in loiter mode

Figure 9. roll in loiter mode

Figure 8 and figure 9 are situation of roll/pitch in loiter mode using the same control law.In loiter mode, fluctuations of roll channel and elevation channel are reduced
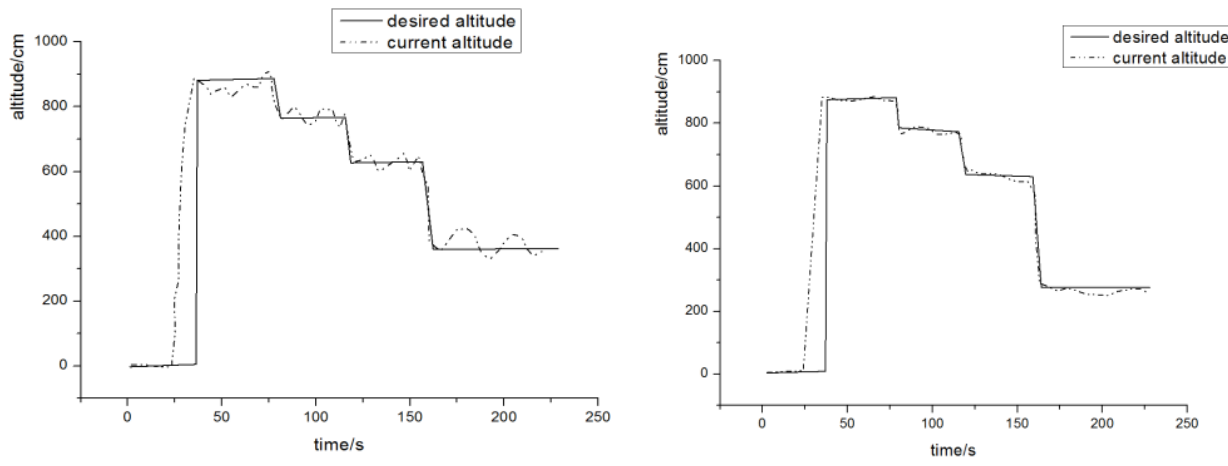

Figure 10. altitude tracking situation before fusion Figure 11. altitude tracking situation after fusion

Figure 10 and figure 11 are situation of altitude tracking. using multi-sensor lead to a more accurate altitude tracking and less shock.

\section{Summary}

The result data shows that multi-sensor fusion method is proposed in this paper is effective, the aircraft can be flight more stably. The future will continue to optimize the algorithm, so that the navigation accuracy is higher.

\section{References}

[1] R.Steven,Swanson.A fuzzy navigational state estimator for GPS/INS intergration. IEEE Symposium of Position Location and Navigation . 1998

[2] Shu-Li Sun.Multi-sensor optimal fusion fixed-interval Kalman smoothers. Information Fusion . 2008

[3] E. B. Song,Y. M. Zhu,J. Zhou.Sensors' optimal dimensionality compression matrix in estimation fusion. Automatica . 2005

[4] Yunmin Zhu,Zhisheng You,Juan Zhao,et al.The optimality for the Distributed Kalman Filtering Fusion with Feedback. Automatica . 2001

[5] E.A.Wan,R.V.D.Merwe.The unscented Kalman filter. Kalman Filtering and Neural Networks . 2001 